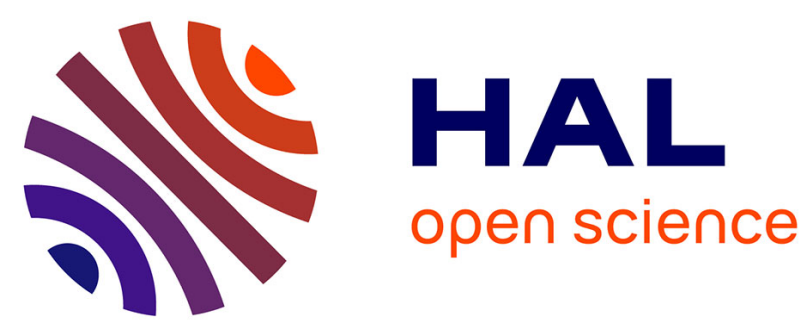

\title{
Facilitation Dynamics and Localization Phenomena in Rydberg Lattice Gases with Position Disorder
}

\author{
Matteo Marcuzzi, Jiří Minar, Daniel Barredo, Sylvain de Léséleuc, Henning
} Labuhn, Thierry Lahaye, Antoine Browaeys, Emanuele Levi, Igor Lesanovsky

\section{- To cite this version:}

Matteo Marcuzzi, Jiří Minar, Daniel Barredo, Sylvain de Léséleuc, Henning Labuhn, et al.. Facilitation Dynamics and Localization Phenomena in Rydberg Lattice Gases with Position Disorder. Physical Review Letters, 2017, 118 (6), pp.063606. 10.1103/PhysRevLett.118.063606 • hal-01615046

\author{
HAL Id: hal-01615046 \\ https://hal.science/hal-01615046
}

Submitted on 11 Oct 2017

HAL is a multi-disciplinary open access archive for the deposit and dissemination of scientific research documents, whether they are published or not. The documents may come from teaching and research institutions in France or abroad, or from public or private research centers.
L'archive ouverte pluridisciplinaire HAL, est destinée au dépôt et à la diffusion de documents scientifiques de niveau recherche, publiés ou non, émanant des établissements d'enseignement et de recherche français ou étrangers, des laboratoires publics ou privés. 


\title{
Facilitation Dynamics and Localization Phenomena in Rydberg Lattice Gases with Position Disorder
}

\author{
Matteo Marcuzzi,,${ }^{1,2}$ Jiří Mináŕ, ${ }^{1,2}$ Daniel Barredo, ${ }^{3}$ Sylvain de Léséleuc, ${ }^{3}$ Henning Labuhn, ${ }^{3}$ \\ Thierry Lahaye, ${ }^{3}$ Antoine Browaeys, ${ }^{3}$ Emanuele Levi, ${ }^{1,2}$ and Igor Lesanovsky ${ }^{1,2}$ \\ ${ }^{1}$ School of Physics and Astronomy, University of Nottingham, Nottingham NG7 2RD, United Kingdom \\ ${ }^{2}$ Centre for the Mathematics and Theoretical Physics of Quantum Non-equilibrium Systems, \\ University of Nottingham, Nottingham NG7 2RD, United Kingdom \\ ${ }^{3}$ Laboratoire Charles Fabry, Institut d'Optique Graduate School, CNRS, \\ Université Paris-Saclay, 91127 Palaiseau cedex, France \\ (Received 5 October 2016; published 10 February 2017)
}

\begin{abstract}
We explore the dynamics of Rydberg excitations in an optical tweezer array under antiblockade (or facilitation) conditions. Because of the finite temperature the atomic positions are randomly spread, an effect that leads to quenched correlated disorder in the interatomic interaction strengths. This drastically affects the facilitation dynamics as we demonstrate experimentally on the elementary example of two atoms. To shed light on the role of disorder in a many-body setting we show that here the dynamics is governed by an Anderson-Fock model, i.e., an Anderson model formulated on a lattice with sites corresponding to many-body Fock states. We first consider a one-dimensional atom chain in a limit that is described by a one-dimensional Anderson-Fock model with disorder on every other site, featuring both localized and delocalized states. We then illustrate the effect of disorder experimentally in a situation in which the system maps on a two-dimensional Anderson-Fock model on a trimmed square lattice. We observe a clear suppression of excitation propagation, which we ascribe to the localization of the manybody wave functions in Hilbert space.
\end{abstract}

DOI: 10.1103/PhysRevLett.118.063606

Introduction.-Rydberg gases provide a versatile platform for studies of quantum few-body and many-body phenomena with applications ranging from quantum information processing [1] to simulations of complex condensed matter systems. The experimental degree of control has reached a stage which enables efficient entanglement creation [2] and implementation of quantum Ising models $[3,4]$. This opens pathways towards probing magnetic structures [5-8] as well as the exploration of open many-body quantum systems [9-15].

Of particular interest is the so-called facilitation mechanism (or antiblockade), where the excitation of an atom to a Rydberg state is strongly enhanced in the vicinity of an already excited atom [16,17]. This effect is of broad relevance and exploited in the design of quantum gates $[18,19]$, as well as in protocols for dissipative quantum state preparation [6]. In the many-body context it effectuates an aggregation mechanism, where an initial Rydberg excitation seed triggers a dynamical growth of excitation clusters [18,20-23] and it enables the implementation of kinetic constraints $[12,24,25]$ thereby connecting to the physics of glass-forming substances [26-28].

Here we perform a theoretical and experimental study of the facilitated dynamics of Rydberg excitations in a onedimensional array of optical tweezers. In a first experiment conducted with only two of them, we establish that the uncertainty of the atomic positions introduces disorder which strongly affects excitation transfer between the atoms. To gain insight on how disorder affects the many-body context we theoretically consider first a regime of small disorder and strong interaction, which lends itself to a description through a one-dimensional Anderson model [29-31] defined on a reduced Hilbert space. Here, the disorder occurs on every other "site" and the corresponding amplitudes are correlated due to their dependence on the interatomic distances. Finally, we conduct an experiment where we probe the excitation dynamics in a linear array of eight tweezers and provide first evidence of a strong suppression of excitation propagation. We show that in the accessed parameter regime the physics is governed by an effective two-dimensional Anderson model on a trimmed square lattice, and we interpret the absence of propagation in terms of localization of the wave function in the Hilbert space.

Rydberg lattice gas with disorder.-We consider a chain of tight optical traps (tweezers), each loaded with a single atom [4,32-34]. Figure 1(a) displays an example for two atoms. We label the Cartesian coordinates with an index $i=1,2,3$, with the chain lying along direction 3 . The average separation between contiguous traps is $\mathbf{r}_{0}=\left(0,0, r_{0}\right)$. We describe the Rydberg atoms as effective two-level systems [35] with the electronic ground state $|\downarrow\rangle$ and a Rydberg excited state (or "excitation") $|\uparrow\rangle$. In the following, we refer to the tensor products of $|\uparrow\rangle$ and $|\downarrow\rangle$ 

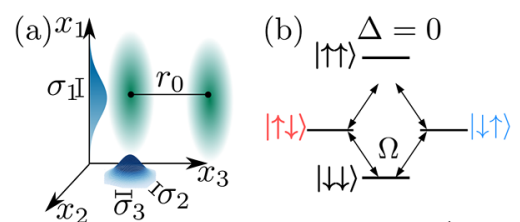

(c) $\Delta=-V_{\mathrm{NN}}$

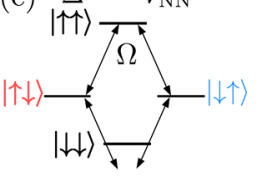

(d) $\quad \Delta=0$

$P_{\downarrow \uparrow} P_{\uparrow \downarrow}{ }^{1}(\mathrm{e})$

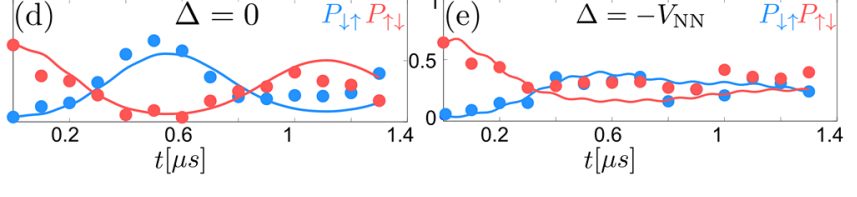

FIG. 1. Two-atom setting. (a) The harmonic traps are disposed in a line along $i=3$ with average separation $r_{0}$ and widths $\sigma_{i}$. (b), (c) Level structure for the two-atom case for the resonant $(\Delta=0)$ and facilitated $\left(\Delta=-V_{\mathrm{NN}}\right)$ conditions, respectively. The experimental data for the time evolution of the excitation probabilities $P_{\uparrow \downarrow}, P_{\downarrow \uparrow}$ are shown as full circles in panels (d),(e). The data are averaged over at least 100 realizations of the disorder. The solid lines show numerical solutions of the dynamics obtained averaging over 30 realizations of the disorder. The experimental data here and in the following were obtained using ${ }^{87} \mathrm{Rb}$ atoms held at a temperature $T=50 \mu \mathrm{K}$ in the traps with frequencies $\omega_{1}=2 \pi \times 11, \omega_{2,3}=2 \pi \times 91.5 \mathrm{kHz}$ resulting in the position uncertainties $\sigma_{1}=1 \mu \mathrm{m}$ and $\sigma_{2,3}=120 \mathrm{~nm}$. The internal levels are $|\downarrow\rangle=\left|5 S_{1 / 2}, F=2, M=2\right\rangle$ and $|\uparrow\rangle=\left|100 D_{3 / 2}, F=3, M=3\right\rangle$ with $r_{0}=14.2 \mu \mathrm{m}, \Omega=2 \pi \times 1.25 \mathrm{MHz}, \quad C_{6}=-2 \pi \times 7.3 \times$ $10^{7} \mathrm{MHz} \mu \mathrm{m}^{6}$. Consequently, $V_{\mathrm{NN}}=C_{6} / r_{0}^{6}=-2 \pi \times 8.9$ and $|\delta V| \sim 2 \pi \times 0.64 \mathrm{MHz}$ (all energies are in units of $\hbar$ ).

states as the "Fock basis." The atoms are driven by laser light with Rabi frequency $\Omega$, and detuning $\Delta$. Excited ones interact via a van der Waals potential $V(|\mathbf{r}|)=C_{6} /|\mathbf{r}|^{6}$ $[35,36]$. The Hamiltonian of the system reads

$$
H=\sum_{k}\left(\frac{\Omega}{2} \sigma_{k}^{x}+\Delta n_{k}+\sum_{l>k} V\left(\left|\mathbf{r}_{k}-\mathbf{r}_{l}\right|\right) n_{k} n_{l}\right),
$$

where $k, l$ are lattice indices, $\sigma_{k}^{x}=\left|\uparrow_{k}\right\rangle\left\langle\downarrow_{k}|+| \downarrow_{k}\right\rangle\left\langle\uparrow_{k}\right|$ and $n_{k}=\left|\uparrow_{k}\right\rangle\left\langle\uparrow_{k}\right|$. We express the $k$ th atom position as $\mathbf{r}_{k}=(k-1) \mathbf{r}_{0}+\delta \mathbf{r}_{k}$. The displacements $\delta \mathbf{r}_{k}$ originate from the finite temperature $T$ of the atoms and constitute an intrinsic source of randomness. For sufficiently low $T$, the atoms, which are frozen during the experiment, occupy the harmonic part of the traps (with frequencies $\omega_{i}$ along $i=1,2,3)$. Hence, their distribution is approximately a Gaussian with widths $\sigma_{i}=\sqrt{k_{B} T /\left(m \omega_{i}^{2}\right)}$, with $m$ the atomic mass [37]. Randomness enters Eq. (1) via the interaction term, which depends on the distances $d_{k, l}=$ $\left|\mathbf{r}_{k+l}-\mathbf{r}_{k}\right|=\left|l \mathbf{r}_{0}+\delta \mathbf{r}_{k+l}-\delta \mathbf{r}_{k}\right|$. Correspondingly, we introduce the energy displacements $\delta V_{k} \equiv V\left(d_{k, 1}\right)-V\left(r_{0}\right)$. Note that these distances are not independently distributed: for instance, $d_{k+1,1}$ and $d_{k, 1}$ both depend on $\mathbf{r}_{k+1}$, which generates correlation between them [37].

Two-atom dynamics and facilitation mechanism.-We begin by illustrating the effect of the disorder in a two-atom setting. Considering first $\Delta=0$ [see Fig. 1(b)], the two (a)
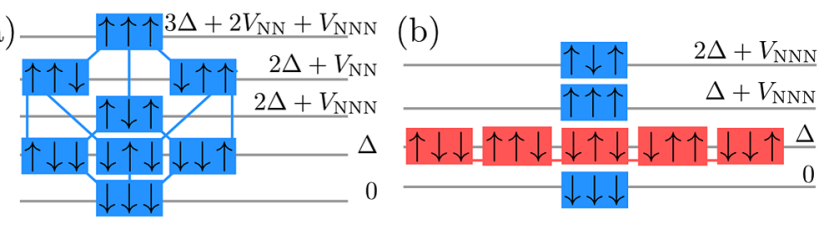

FIG. 2. Fock space structure for three atoms prior and after applying the facilitation condition. States with similar energy occupy the same row. (a) Internal structure of the Fock space. Linked states are connected by one spin flip. (b) Fock states spanning the reduced Hilbert space under facilitation conditions are shown in red (see text for details).

atomic states $|\uparrow \downarrow\rangle,|\downarrow \uparrow\rangle$ are resonant with $|\downarrow \downarrow\rangle$, while the interaction brings the state $|\uparrow \uparrow\rangle$ off resonance and thus decouples it from the dynamics. Since the disorder only acts on $|\uparrow \uparrow\rangle$, a dynamics starting from $|\downarrow \downarrow\rangle,|\uparrow \downarrow\rangle,|\downarrow \uparrow\rangle$, or combinations thereof, is not affected by it. In the experiment, after preparing the system in the $|\uparrow \downarrow\rangle$ state, the evolution thus resembles a coherent oscillation of the initial excitation between the atoms. This is shown in Fig. 1(d), where we display the excitation probabilities $P_{\uparrow \downarrow}=$ $\left\langle n_{1}\left(1-n_{2}\right)\right\rangle, P_{\downarrow \uparrow}=\left\langle\left(1-n_{1}\right) n_{2}\right\rangle$ as functions of time. The presence of the disorder becomes apparent instead when driving the system through the $|\uparrow \uparrow\rangle$ resonance. This is achieved by setting $\Delta=-V_{\mathrm{NN}}$ [Fig. 1(c)], the so-called "facilitation condition" [24,43-46], where $V_{\mathrm{NN}}=V\left(r_{0}\right)$ is the nearest-neighbor interaction energy in the absence of disorder. Here, the amplitude of the oscillations of $P_{\downarrow \uparrow}$ and $P_{\uparrow \downarrow}$ is suppressed, see Fig. 1(e). This means that the displacements $\delta \mathbf{r}_{1}, \delta \mathbf{r}_{2}$ can be sufficiently large to bring the $|\uparrow \uparrow\rangle$ state off-resonance, hindering in turn the propagation of the initial excitation. Note that the initial state $|\uparrow \downarrow\rangle$ is obtained with nonunit probability due to experimental imperfections (see Supplemental Material [37] for details). We also refer to Refs. [4,33] for further details on the experimental setting.

Generalization to many atoms.-We investigate now the facilitated propagation of an excitation through a onedimensional chain of atoms and first consider a simplified situation before addressing the parameter regime accessed by our experiment. The Hilbert space of our system can be depicted as a complex network of Fock states. Only states which differ by a single spin flip are connected by Hamiltonian (1) via the "flipping" $(\propto \Omega)$ term. This is sketched in Fig. 2(a) for three atoms, where we label the states with their diagonal energies (i.e., their energies for $\Omega=0)$, dub $V_{\mathrm{NNN}}=V\left(2 r_{0}\right)$ the next-nearest-neighbor interactions and assume we can neglect all terms $V\left(n r_{0}\right)$ for $n>2$. In the following, we fix the facilitation condition $\Delta=-V_{\mathrm{NN}}$ and we make a number of simplifying assumptions: (i) large detuning $(\Delta \gg \Omega$ ). This strongly suppresses unfacilitated transitions; (ii) strong next-nearest-neighbor blockade $\left[V\left(2 r_{0}\right) \gg \Omega, \delta V_{k}\right]$. Interactions at distance $2 r_{0}$ are supposed to be sufficiently strong to suppress 
transitions. In particular, we require this suppression to be much stronger than the one produced by the disorder. We also consider a tight confinement of the atoms, $\sigma_{j} \ll r_{0}$, such that, as in Fig. 1(e), the disorder can hinder, but not prevent transport entirely (i.e., $\delta V_{k} \lesssim \Omega$ ).

Under these conditions the states organize in layers with large energy gaps approximately of the order of $V_{\mathrm{NNN}}$ or $\Delta$. Within each layer, however, states are now separated by considerably smaller differences $\delta V_{k}$. We thereby neglect connections between different layers and retain only the intralayer ones [see Figs. 2(a),2(b)].

We focus now on the highlighted (red) layer at energy $\Delta$ [Fig. 2(b)]. We recall first that (i) implies that spins cannot be flipped if they do not have a single excited neighbor. As a consequence, clusters of consecutive excitations can shrink or grow, but not merge or (dis)appear; i.e., the number $N_{\mathrm{cl}}$ of these clusters is conserved (see also the discussion in Ref. [37]). Condition (ii) implies instead that a spin next to two consecutive excitations cannot flip (e.g., $|\uparrow \uparrow \downarrow\rangle \leftrightarrow|\uparrow \uparrow \uparrow\rangle$ is forbidden); it then follows that the number $N_{\mathrm{NNN}}$ of excitation triples ( $\uparrow \uparrow$ ) is conserved. The red layer in Fig. 2(b) corresponds to $N_{\mathrm{cl}}=1, N_{\mathrm{NNN}}=0$ as it exclusively includes states with a single excitation or a single pair of neighboring ones; in the following, the former kind will be denoted by odd integers, $|2 j-1\rangle \equiv$ $\left|\downarrow_{1} \ldots \downarrow_{j-1} \uparrow_{j} \downarrow_{j+1} \ldots \downarrow_{L}\right\rangle \quad(j=1 \ldots L)$, whereas the latter by even integers, $\quad|2 j\rangle \equiv\left|\downarrow_{1} \ldots \downarrow_{j-1} \uparrow_{j} \uparrow_{j+1} \downarrow_{j+2} \ldots \downarrow_{L}\right\rangle$ $(j=1 \ldots L-1)$. The dynamics restricted to this layer can be described by a one-dimensional Anderson model [29]. In fact, the Hamiltonian connects these states sequentially $(\ldots|2 j-1\rangle \leftrightarrow|2 j\rangle \leftrightarrow|2 j+1\rangle \ldots)$, taking the form of a tight-binding model with sites labeled by $b=1 \ldots 2 L-1$ and a random potential $h_{b}=\left[1+(-1)^{b}\right] \delta V_{b / 2} / \Omega$ acting only on even ones. In this restricted space $H$ can be recast as [37]

$H_{\mathrm{A}}=\frac{\Omega}{2} \sum_{b=1}^{2 L-2}\left(|b\rangle\langle b+1|+| b+1\rangle\left\langle b\left|+h_{b}\right| b\right\rangle\langle b|\right)$.

The two main differences to the "canonical" Anderson model lie in the absence of disorder on odd sites and the fact that the $h_{b}$ are identically distributed, but not independent random variables. In order to distinguish it from a standard Anderson model defined in real space, i.e., on a physical lattice, in the following we shall call it the Anderson-Fock model.

Localization in the 1D Anderson-Fock model.-Our analysis-analogously to the canonical case-focuses on the eigenvectors $\left|\psi_{n}\right\rangle$ of $H_{A}$. In the Fock basis, we distinguish between localized states whose amplitude $\left|\left\langle b \mid \psi_{n}\right\rangle\right|$ is concentrated within a region of width $l$ and decays exponentially $\sim \mathrm{e}^{-r / l}$ with the distance $r$ from it, and delocalized states which are instead extended and do not show exponentially suppressed tails. Equivalently, one can introduce the Lyapunov exponent $\gamma=l^{-1}[38,39]$. Wave

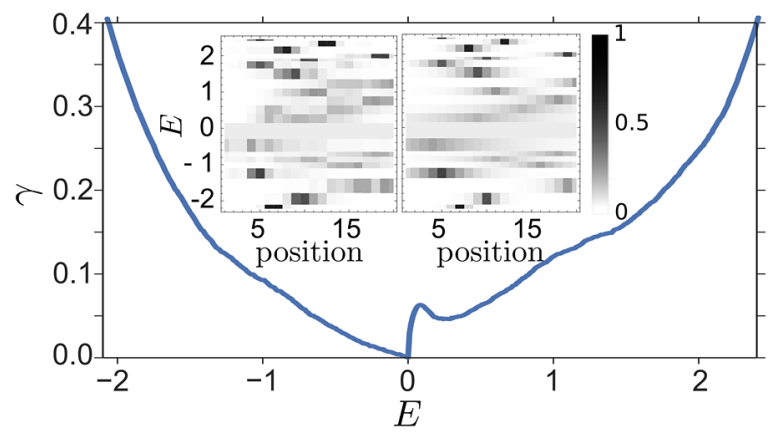

FIG. 3. Lyapunov exponent for the one-dimensional AndersonFock model. All data shown in this figure are obtained with the same parameters given in Fig. 1. In the main figure we report the Lyapunov exponent as a function of the energy $E$ (measured in units of $\Omega / 2$ ). The inset shows a comparison between the shapes of the wave functions obtained from a numerical reconstruction (left panel) and from the corresponding prediction associated to the Lyapunov exponent (right panel) for a chain of $L=20$ sites and a specific realization of the disorder. In the right panel the envelopes $\propto \exp \left[-4 \gamma(E)\left|k-k_{\max }(E)\right|\right]$ are centered at the position $k_{\max }(E)$ at which the corresponding set of excitation probabilities in the left panel reaches its maximum. The factor 4 in the exponent stems from considering probabilities instead of amplitudes and the fact that the length of the atomic chain is about half the one in Fock space.

functions with $\gamma>0$ are localized, whereas $\gamma=0$ denotes delocalization. We emphasize that the Lyapunov exponent, as a function of the energy $E$, only depends on the distribution of the disorder, and not on the specific realizations thereof [40]. In Fig. 3 we report a numerical determination of $\gamma$ for a chain of length $L=25000$ sites. We provide details of these computations in Ref. [37]. We find that $\gamma$ is positive $\forall E \neq 0$, while $\gamma(E=0)=0$, signaling the presence of a delocalized state. The asymmetric shape originates from an asymmetry of the distribution of energy displacements between positive and negative values [37]. In the inset we compare our Lyapunov exponent results with a numerical simulation of a system of size $L=20$ (for a randomly chosen realization of the disorder). This shows that the Lyapunov exponent provides a reasonably reliable prediction already for relatively small system sizes.

Note, that $E=0$ is always-independently of the realization of disorder-an eigenvalue of $H_{\mathrm{A}}$ and corresponds to the (delocalized) wave function $\left|\psi_{0}\right\rangle=$ $(1 / \sqrt{L}) \sum_{b} \sin (\pi b / 2)|b\rangle$, which has nonvanishing components only on states not affected by the disorder. This is in contrast to the standard Anderson model [29], which features full localization, and is instead reminiscent of related works on one dimensional models: the random dimer model $[38,47-50]$ and the Anderson model in the presence of correlated disorder [39], both exhibiting delocalized states in the spectrum.

Localization in the 2D Anderson-Fock model.-Moving away from this simplified discussion we now analyze an 


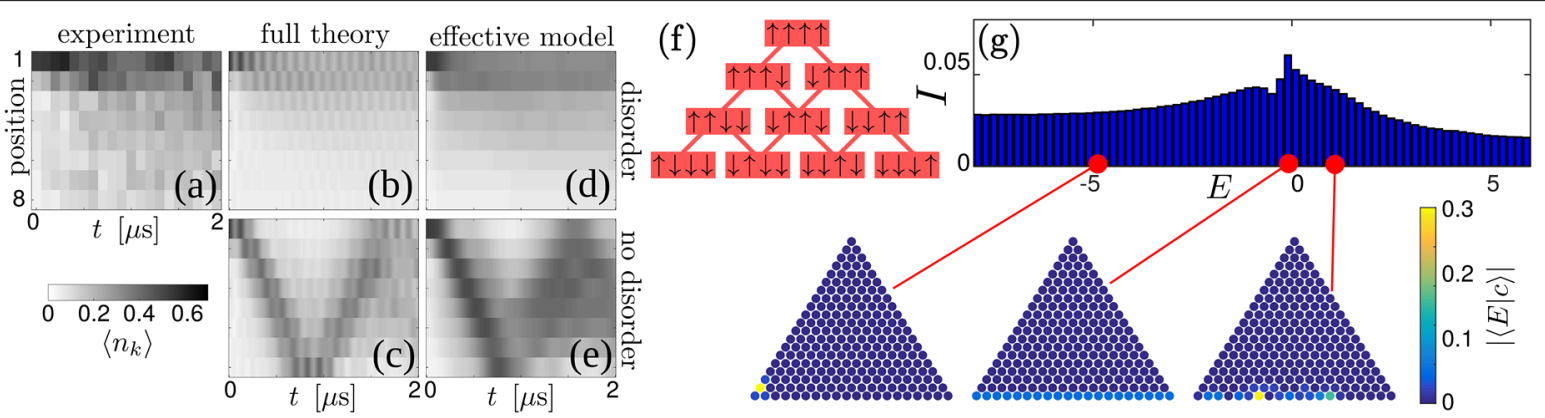

FIG. 4. Eight-atom experiment and two-dimensional Anderson-Fock model. (a) Experimental data for the dynamics of the site-resolved excitation probability averaged over more than 100 realizations. Here, $|\uparrow\rangle=\left|56 D_{3 / 2}, F=3, M=3\right\rangle, r_{0}=4.1 \mu \mathrm{m}$; $\Omega=2 \pi \times 2.1, \Delta=-V_{\mathrm{NN}}=-2 \pi \times 8.4$, and $|\delta V| \sim 2 \pi \times 2.1 \mathrm{MHz}$ (in units of $\hbar$ ). The data are compared with numerical data from exact diagonalization of the Hamiltonian (b),(c), and the 2D Anderson-Fock model (d),(e), with and without disorder. Disorder averages are made over 100 realizations. In the absence and for low disorder $(|\delta V| \lesssim 2 \pi \times 0.4 \mathrm{MHz})$, excitations still propagate ballistically. (f) Lattice structure of the effective model for $L=4$ atoms and $N_{\mathrm{cl}}=1$. (g) Inverse participation ratio $I$ as a function of the energy $E$ (measured in units of $\Omega / 2$ ) for a chain of $L=20$ atoms. The amplitude of the wave function (projecting $|E\rangle$ on the Fock basis $|c\rangle$ ) is reported for four representative states on a lattice whose structure follows the one shown in panel (f). From left to right they display: a state localized in both Fock space and real space, the special state $\left|\psi_{0}\right\rangle$ and a similar state found for small $E>0$ (see text for details).

experiment conducted in a chain of 8 atoms. We monitor the local densities $\left\langle n_{k}(t)\right\rangle$ starting from a single excitation $\left|\psi_{\text {in }}\right\rangle=|\uparrow \downarrow \downarrow \downarrow \downarrow \downarrow \downarrow \downarrow\rangle$. The result is displayed in Fig. 4(a) and no appreciable propagation beyond the second site is observed, signaling a strong suppression of transport. As shown in the following, this can again be ascribed to the fact that the eigenstates are localized, although via a slightly modified theoretical description.

Solving Hamiltonian (1) in the presence of disorder, Fig. 4(b), we find localization, in good agreement with the experimental data. The case without randomness, studied only numerically [Fig. 4(c)], features instead propagation. In our experiment, condition (ii) (i.e., $V_{\mathrm{NNN}} \gg \delta V_{k}$ ) is not satisfied. It is thereby possible to grow clusters beyond the two-excitation limit. This breaks the chainlike structure obtained from the simplified description [Fig. 2(b)]. Instead it gives rise-when considering a single cluster $\left(N_{\mathrm{cl}}=1\right)-$ to a two-dimensional square lattice with $N=L(L+1) / 2$ sites. This structure, previously discussed in Ref. [41], is sketched in Fig. 4(f) for four atoms. We remark that the two bottommost rows correspond precisely to the previous onedimensional chain. The dynamics on this "triangle" of states is described by a 2D tight-binding Anderson-Fock model similar to Eq. (2) (see Ref. [37] for the derivation). As shown in Figs. 4(d)-4(e) the solutions of this effective model agree well with that of the full Hamiltonian.

The observed inhibition of excitation propagation stems from the localization of the many-body eigenstates $|E\rangle$ on the restricted Fock basis $|c\rangle$. We quantify the degree of localization by means of the inverse participation ratio (IPR) $I=\left(N \sum_{c}|\langle E \mid c\rangle|^{4}\right)^{-1} \quad$ [51]. As a measure of localization, the IPR can be easily tested on the two limiting cases: for a state $|E\rangle$ uniformly distributed on the basis $(|\langle E \mid c\rangle|=1 / \sqrt{N})$ one finds the maximal value $I=1$, whereas for a completely localized state, namely,
$|E\rangle \equiv|\bar{c}\rangle$ corresponding to a single Fock state $|\bar{c}\rangle$, one has $I=1 / N$. A numerical study of $I$ for $L=20$ atoms and the parameter set employed in the experiment is reported in Fig. 4(g), where for every realization of the disorder the spectrum is calculated via exact diagonalization. The IPR is then computed for each energy eigenvector and a first average is calculated among levels which end up in the same bin of the histogram. A second average is then applied over all the considered realizations. In general, we observe that the IPR remains rather low on the entire spectrum $(I<0.1)$, signaling that the parameters are in the localized phase. The form of the IPR indicates the presence of strongly localized states at large energies (both positive and negative), while eigenstates at smaller energies are slightly more spread-out. The central peak links to the presence of the state $\left|\psi_{0}\right\rangle=|E=0\rangle$ encountered above, which is still an exact eigenstate, but only occupies the bottommost row [see example in Fig. 4(g)], its IPR being $I=L / N=2 /(L+1)$.

It is important to remark that despite the mapping onto a single particle Anderson problem in Fock space, the system is in fact interacting in real space and does not reduce, in general, to a noninteracting Anderson problem. In particular, rephrasing the dynamics in terms of domain wall degrees of freedom [52,53] does not yield free particles [37].

Outlook.-The Rydberg excitation dynamics in detuned optical tweezer arrays is governed by certain classes of tight binding Anderson models featuring inhibited excitation transport, the simplest one being a 1D Anderson-Fock model with disorder on every other site. Currently accessed experimental parameter regimes feature a 2D manifestation of an Anderson-Fock model with correlated disorder, whose behavior is largely unexplored. The presented system opens possibilities for studies of multidimensional Anderson models, where the dimensionality is twice the 
number of excitation clusters. This connection may shed light on how Fock space localization influences real space localization, which is a subtle and interesting open problem in the context of many-body localization [54,55], which started to be addressed experimentally only very recently $[56,57]$.

I. L. thanks Juan P. Garrahan for fruitful discussions. The research leading to these results has received funding from the European Research Council under the European Union's Seventh Framework Programme (FP7/20072013)/ERC Grant Agreement No. 335266 (ESCQUMA), the EU-FET Grant No. 512862 (HAIRS), the H2020FETPROACT-2014 Grant No. 640378 (RYSQ), and EPSRC Grant No. EP/M014266/1 and by the Région Ile-de-France in the framework of DIM Nano-K.

[1] M. Saffman, T. G. Walker, and K. Mølmer, Rev. Mod. Phys. 82, 2313 (2010).

[2] Y.-Y. Jau, A. Hankin, T. Keating, I. Deutsch, and G. Biedermann, Nat. Phys. 12, 71 (2016).

[3] P. Schauß, J. Zeiher, T. Fukuhara, S. Hild, M. Cheneau, T. Macrì, T. Pohl, I. Bloch, and C. Groß, Science 347, 1455 (2015).

[4] H. Labuhn, D. Barredo, S. Ravets, S. de Léséleuc, T. Macrì, T. Lahaye, and A. Browaeys, Nature (London) 534, 667 (2016).

[5] T. E. Lee, H. Häffner, and M. C. Cross, Phys. Rev. A 84, 031402 (2011).

[6] A. W. Carr and M. Saffman, Phys. Rev. Lett. 111, 033607 (2013).

[7] M. Hoening, W. Abdussalam, M. Fleischhauer, and T. Pohl, Phys. Rev. A 90, 021603 (2014).

[8] R. M. W. van Bijnen and T. Pohl, Phys. Rev. Lett. 114, 243002 (2015).

[9] H. Weimer, Phys. Rev. A 91, 063401 (2015).

[10] M. Marcuzzi, M. Buchhold, S. Diehl, and I. Lesanovsky, Phys. Rev. Lett. 116, 245701 (2016).

[11] D. C. Rose, K. Macieszczak, I. Lesanovsky, and J. P. Garrahan, Phys. Rev. E 94, 052132 (2016).

[12] B. Everest, M. Marcuzzi, J. Garrahan, and I. Lesanovsky, Phys. Rev. E 94, 052108 (2016).

[13] V. R. Overbeck, M. F. Maghrebi, A. V. Gorshkov, and H. Weimer, arXiv:1606.08863.

[14] N. Šibalić, C. G. Wade, C. S. Adams, K. J. Weatherill, and T. Pohl, Phys. Rev. A 94, 011401 (2016).

[15] S. Helmrich, A. Arias, and S. Whitlock, arXiv:1605.08609.

[16] C. Ates, T. Pohl, T. Pattard, and J. M. Rost, Phys. Rev. Lett. 98, 023002 (2007).

[17] T. Amthor, C. Giese, C. S. Hofmann, and M. Weidemüller, Phys. Rev. Lett. 104, 013001 (2010).

[18] M. Gärttner, K. P. Heeg, T. Gasenzer, and J. Evers, Phys. Rev. A 88, 043410 (2013).

[19] R. C. Teixeira, C. Hermann-Avigliano, T. L. Nguyen, T. Cantat-Moltrecht, J. M. Raimond, S. Haroche, S. Gleyzes, and M. Brune, Phys. Rev. Lett. 115, 013001 (2015).

[20] I. Lesanovsky and J. P. Garrahan, Phys. Rev. A 90, 011603 (2014).
[21] H. Schempp, G. Günter, M. Robert-de Saint-Vincent, C. S. Hofmann, D. Breyel, A. Komnik, D. W. Schönleber, M. Gärttner, J. Evers, S. Whitlock, and M. Weidemüller, Phys. Rev. Lett. 112, 013002 (2014).

[22] A. Urvoy, F. Ripka, I. Lesanovsky, D. Booth, J. P. Shaffer, T. Pfau, and R. Löw, Phys. Rev. Lett. 114, 203002 (2015).

[23] C. Simonelli, M. M. Valado, G. Masella, L. Asteria, E. Arimondo, D. Ciampini, and O. Morsch, J. Phys. B: At. Mol. Opt. Phys. 49, 154002 (2016).

[24] I. Lesanovsky and J. P. Garrahan, Phys. Rev. Lett. 111, 215305 (2013)

[25] M. M. Valado, C. Simonelli, M. D. Hoogerland, I. Lesanovsky, J. P. Garrahan, E. Arimondo, D. Ciampini, and O. Morsch, Phys. Rev. A 93, 040701 (2016).

[26] J. P. Garrahan and D. Chandler, Proc. Natl. Acad. Sci. U.S.A. 100, 9710 (2003).

[27] J. P. Garrahan, P. Sollich, and C. Toninelli, arXiv:1009.6113.

[28] G. Biroli and J. P. Garrahan, J. Chem. Phys. 138, 12A301 (2013).

[29] P. W. Anderson, Phys. Rev. 109, 1492 (1958).

[30] N. Mott and W. Twose, Adv. Phys. 10, 107 (1961).

[31] K. Ishii, Prog. Theor. Phys., Suppl. 53, 77 (1973).

[32] F. Nogrette, H. Labuhn, S. Ravets, D. Barredo, L. Béguin, A. Vernier, T. Lahaye, and A. Browaeys, Phys. Rev. X 4, 021034 (2014).

[33] H. Labuhn, S. Ravets, D. Barredo, L. Béguin, F. Nogrette, T. Lahaye, and A. Browaeys, Phys. Rev. A 90, 023415 (2014).

[34] D. Barredo, H. Labuhn, S. Ravets, T. Lahaye, A. Browaeys, and C. S. Adams, Phys. Rev. Lett. 114, 113002 (2015).

[35] R. Löw, H. Weimer, J. Nipper, J. B. Balewski, B. Butscher, H. P. Büchler, and T. Pfau, J. Phys. B 45, 113001 (2012).

[36] L. Béguin, A. Vernier, R. Chicireanu, T. Lahaye, and A. Browaeys, Phys. Rev. Lett. 110, 263201 (2013).

[37] See Supplemental Material at http://link.aps.org/ supplemental/10.1103/PhysRevLett.118.063606 for details of the disorder description, derivation of the effective Hamiltonians and numerical simulations, which includes Refs. [38-42].

[38] F. M. Izrailev, T. Kottos, and G. P. Tsironis, Phys. Rev. B 52, 3274 (1995).

[39] F. M. Izrailev and A. A. Krokhin, Phys. Rev. Lett. 82, 4062 (1999).

[40] H. Furstenberg and H. Kesten, Ann. Math. Stat. 31, 457 (1960).

[41] M. Mattioli, A. W. Glätzle, and W. Lechner, New J. Phys. 17, 113039 (2015).

[42] C. da Fonseca and J. Petronilho, Linear Algebra Appl. 325, 7 (2001).

[43] C. Ates, T. Pohl, T. Pattard, and J. M. Rost, Phys. Rev. Lett. 98, 023002 (2007).

[44] T. Amthor, C. Giese, C. S. Hofmann, and M. Weidemüller, Phys. Rev. Lett. 104, 013001 (2010).

[45] I. Lesanovsky and J. P. Garrahan, Phys. Rev. A 90, 011603 (2014).

[46] M. M. Valado, C. Simonelli, M. D. Hoogerland, I. Lesanovsky, J. P. Garrahan, E. Arimondo, D. Ciampini, and O. Morsch, Phys. Rev. A 93, 040701 (2016).

[47] J. C. Flores, J. Phys. Condens. Matter 1, 8471 (1989). 
[48] D. H. Dunlap, H.-L. Wu, and P. W. Phillips, Phys. Rev. Lett. 65, 88 (1990).

[49] A. Bovier, J. Phys. A 25, 1021 (1992).

[50] S. De Bièvre and F. Germinet, J. Stat. Phys. 98, 1135 (2000).

[51] R. J. Bell and P. Dean, Discuss. Faraday Soc. 50, 55 (1970).

[52] J.-S. Lee and A. K. Khitrin, Phys. Rev. A 71, 062338 (2005).

[53] G. B. Furman, S. D. Goren, J.-S. Lee, A. K. Khitrin, V. M. Meerovich, and V. L. Sokolovsky, Phys. Rev. B 74, 054404 (2006).
[54] I. V. Gornyi, A. D. Mirlin, and D. G. Polyakov, Phys. Rev. Lett. 95, 206603 (2005).

[55] D. Basko, I. Aleiner, and B. Altshuler, Ann. Phys. (Amsterdam) 321, 1126 (2006).

[56] M. Schreiber, S. S. Hodgman, P. Bordia, H. P. Lüschen, M. H. Fischer, R. Vosk, E. Altman, U. Schneider, and I. Bloch, Science 349, 842 (2015).

[57] J. Smith, A. Lee, P. Richerme, B. Neyenhuis, P. W. Hess, P. Hauke, M. Heyl, D. A. Huse, and C. Monroe, Nat. Phys. 12, 907 (2016). 